\title{
Survey on Hierarchical Cluster Routing Protocols of WSN
}

\author{
Jaspinder Kaur \\ M.Tech Student \\ Computer Science Department, CTIEMT \\ Shahpur, Jalandhar, India
}

\begin{abstract}
Approaches in wireless sensor network (WSN) technology has gained the availability of small and low-cost sensor nodes with sensing capability of various types of physical and environmental conditions, data processing, and wireless communication. In wireless sensor networks have conduct to many new protocols particularly designed for sensor networks where energy awareness is an essential issue? Most of the observation, however, has been given to the routing protocols since they just differ depending on the application and network architecture. This paper surveys recent routing protocols for sensor networks and presents a classification [2] for the various approaches pursued. There are three main types data-centric, hierarchical and location-based.the only one type explored in this paper is hierarchical. Each hierarchical routing protocol is described.
\end{abstract}

\section{General Terms}

Micro-electro-mechanical system, Sensors, WSN, Routing Protocols, energy efficiency, is clustering.

\section{Keywords}

WSN, Routing Protocols, nodes, energy.

\section{INTRODUCTION}

Wireless sensor networks (WSNs) have approached worldwide concentration in recent years, especially with the expansion in Micro-Electro-Mechanical Systems (MEMS) technology which [1] has make possible the development of smart sensors. These sensors are small, with restricted processing and computing resources, and they are affordable compared to traditional sensors. These sensor nodes can sense, measure, and gather [1] information from the environment and, based on some local resolution process, they can send the sensed data to the user. Smart sensor nodes are low power devices provide with one or more sensors, a processor, memory, a power supply, a radio, and an actuator [1]. A variety of mechanical, thermal, biological, chemical, optical, and magnetic sensors may [1] be devoted to the sensor node to measure properties of the environment. Since the sensor nodes have restricted memory and are regularly deployed in unreachable locations, a radio is applied for wireless communication to transfer the data to a base station (e.g., a laptop, a personal handheld device, or an access point to a fixed infrastructure) [1]. Battery is the most important power source in a sensor node. Secondary power supply the return power from the environment such as solar panels may be added to the node depending on the correctness of the environment where the sensor will be deployed. Based on the application and the type of sensors used, actuators may be incorporated in the sensors

\author{
Varsha Sahni \\ Assistant professor \\ M.Tech Student \\ Computer Science Department, CTIEMT \\ Shahpur, Jalandhar, India
}

\section{ROUTING PROTOCOLS IN WIRELESS SENSOR NETWORKS}

Routing protocols have vast range in research area when execute in WSN. These protocols can be categorized on the basis of network structure (designed for the application) [5], routing paths fixed, and network operations and as a provoker of communications. The taxonomy of routing protocols which are further partition into subcategories and helpful in designing of network protocol. In this portion, the routing protocols for WSNs are explained.

\subsection{Network structure based Routing Protocols}

Routing protocols pivot on network structure form routing protocols behaves seriously with respect to the design constraints set for the network structure or area. These are in turn categorized as Flat routing (Data Centric), hierarchical routing (Clustering) and Location (Geographic) routing [5]. fig1. Shows the categories of routing protocols [7]

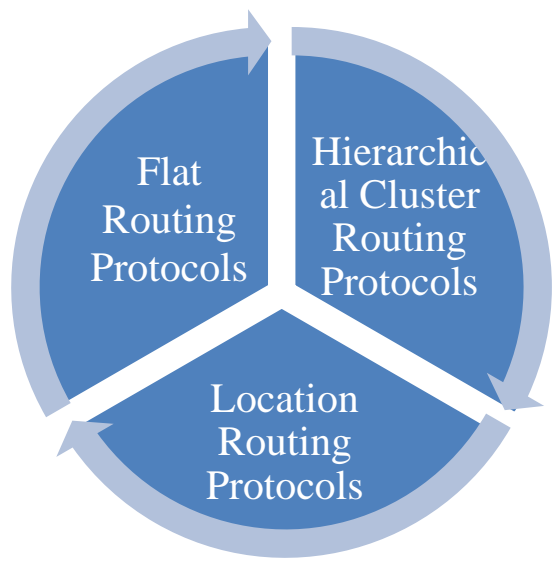

\subsubsection{Flat Routing (Data Centric)}

Data-centric protocols vary from traditional address-centric protocols in the mode that the data is transmitted from source sensors to the base station. In address-centric protocols, each source sensor that has the proper data responds by sending its data to the base station separately of all other sensors. However, in data-centric protocols, when the source sensors transmit their data to the base station, middle sensors can perform some form of aggregation on the data establish from multiple source sensors and send the aggregated data toward the base station. Because of less transmission required to send the data from the sources to the base station this process can outcome in energy savings. In this section, we review some of the data-centric routing protocols for WSNs [11].

\subsubsection{Location Routing (Geographic)}

Practically, in the sensor networks the routing protocols have need of location information for sensor nodes. In most states 
location information is needed in order to measure the distance between two particular nodes so that energy utilization can be calculated. Since, there is no directing scheme for sensor networks like IP-addresses and they are located on a region, location information can be using in routing data in an energy efficient way. The location information is used to locate the position of sensor nodes. To evaluate the nearest neighboring node distance two techniques are used i.e. finding the coordinate of the neighboring node and the other is to use GPS (Global Positioning System).Since, sensor networks are spatially deployed in a region with no addressing scheme, location information can be utilized in routing of data in an energy efficient way.

\subsubsection{Hierarchical Cluster Routing Protocols}

Many research programmers' in the previous few years have consider hierarchical clustering in WSN from different aspects. Clustering is an energy-efficient communication protocol [3] that can be making used by the sensors to broadcast their sensed data to the sink. A test of layered protocol is related with various different clusters of sensors. Every cluster is directed by a special node, called cluster head, which is responsible for arrange the data transmission activities of all sensors in its cluster., a hierarchical approach divided the network into clustered layers .Nodes are grouped into clusters with a cluster head that has the responsibility [3] of routing from one cluster to the other cluster heads or base stations. Data transfer from a lower clustered layer to a higher one. While, it hops from one to another node, but as it hops from one layer to another layer it covers huge distances. This precedes the data faster to the base station. Clustering gives hierarchical-based routing protocols for WSNs. LEACH is one of the clustering hierarchical routing protocols in WSN.

\subsubsection{Low-Energy Adaptive Clustering Hierarchy $(\mathrm{LEACH})$}

LEACH is the initial and most popular energy efficient hierarchical clustering algorithm for WSNs that was developed for decreasing power consumption. In LEACH, the clustering job is rotated between the nodes, depends on duration. Direct communication is used by every Cluster head to send the data to the base station (BS). It is an applicationspecific data distribution protocol that uses clusters to extend the life of the wireless sensor network. LEACH is depends on an aggregation (or fusion) technique that collects or aggregates the original data into a smaller size of data that carry only relevant information to all individual sensors. LEACH divides the network into v cluster several of sensors, which are created by using localized. coordination and control not only to decrease the amount of data that are send to the sink, but also to make routing and data distribution more scalable and durable[3].

Provided that energy dissipation of the sensor based on the distance and the data size to be transferred, LEACH experiments to send data over short distances and reduce the number of transmission and reception processes. The main characteristics of LEACH are: (i) randomized rotation of the Cluster Head and correlated clusters, (ii) local compaction to reduce global communication, (iii) and localized coordination and control for cluster set-up and operation. LEACH uses a casual rotation of high-energy Cluster Head position in place of choosing in static manner, to provide a chance to all sensors to respond as Cluster Heads and avoid the battery consumption of an individual sensor and dying quickly. The process of LEACH is divided into stages, each of which has mainly two phases namely (i) A setup phase is the first phase to manage the network into clusters, Cluster Head advertisement, and transmission schedule creation and

(ii) A steady-state phase is the second phase for data aggregation, compression, and transmission to the Base Station. Cluster heads (CHs) use CSMA MAC protocol to advertise their status [3]. Thus, all non-cluster head sensors must keep their receivers ON during the setup phase [3] in order to hear the announcements sent by the Cluster Heads. These $\mathrm{CHs}$ are chosen with some probability by themselves and broadcast their statuses to the other sensors in the network.

\subsubsection{Enhanced Low-Energy Adaptive \\ ClusteringHierarchy (ELEACH)}

E-LEACH also enhanced LEACH in two major perspectives. E-LEACH advances a cluster head selection algorithm for sensor networks that have non-uniform beginning energy level between the sensors. However, this algorithm considers that sensors have global information about other sensors rest energy. E-LEACH also direct that, under certain expectations, the required number of cluster heads has to scale as the [3] square root of the total number of sensor nodes to minimize the total energy consumption. Other perspectives of ELEACH are the alike LEACH.

\subsubsection{LEACH-Centralized (LEACHC)}

LEACH-C used centralized clustering algorithm and same steady-state protocol. At the time of set-up phase of LEACH$\mathrm{C}$, each node transmits information about current location and energy level to base station (BS). The Base Station will form clusters, Cluster head node and non-Cluster head nodes of each cluster. The Base Station applies its global information of the network to produce good clusters that need less energy for data sending. The number of $\mathrm{CHs}$ in each round of LEACH-C equals a fixed optimal value [3], whereas for $\mathrm{LEACH}$ the number of $\mathrm{CHs}$ differs from round due to the lack of global coordination among nodes.

\subsubsection{Multi-hop LEACH (M-LEACH)}

M-LEACH improves LEACH enable sensor nodes to use multi-hop communication within the cluster to go to increase the energy efficiency of the protocol. Particularly define special nodes (called gateways) that are capable to send the information produce inside the cluster directly to the sink. This work increase the existing results by allocate multi hop inter-cluster communication in thin WSNs in which the direct communication between $\mathrm{CHs}$ or the sink is not feasible due to the distance between them. Thus, the main change of the result proposed here is that the multi-hop approach is followed inside the cluster (sensor nodes to the $\mathrm{CH}$ ) and outside the cluster (from $\mathrm{CHs}$ to the sink using intermediate sensor nodes). $\mathrm{CHs}$ can also execute data fusion to the data receive, allowing a depletion in the total transmitted and redirect data in the network.

\subsubsection{LEACH with Fixed Cluster (LEACH-F)}

LEACH-F is the fresh progress of LEACH, which is depending on clusters that are formed once and then fixed. Then, the $\mathrm{CH}$ pose rotates among the nodes within the cluster. The benefits with this are that, once the clusters are formed, there is no setup load at the development of each round. To decide clusters, LEACH-F uses the same centralized cluster formation algorithm as LEACH-C [3]. The fixed clusters in LEACH-F [3] do not permit new nodes to be added to the system and do not modify their performance based on nodes dying. 


\subsubsection{PEGASIS and Hierarchical-PEGASIS}

PEGASIS is an development of the LEACH protocol[11], which make chains from sensor nodes so that each node sends and receives from a neighbor and to send data to the base station (sink) only one node is chosen from that chain .The data is collected and transfer from node to node, aggregated and finally sent to the base station. The chain creation is executed in a greedy way. Unlike LEACH, PEGASIS stay away from cluster evolution and uses only one node in a chain to send to the BS (sink) rather using multiple nodes. A sensor transmits to its limited neighbors in the head data fusion phase rather sending directly to its Cluster as in the case of LEACH. In PEGASIS routing protocol, the creation stage suppose that all the sensors have global information about the network, especially, the location of the sensors, and use a greedy approach. When a sensor fails or dies due to low battery power [11], the chain is created using the same greedy approach by avoids the failed sensor. In each round, an irregular selected sensor node from the chain will transfer the aggregated data to the BS, thus decrease the per round energy spending compared to LEACH. PEGASIS have ability to increase the lifetime [11] of the network twice as much the lifetime of the network under the LEACH protocol. Such performance gain is achieved by the elimination of the load caused by dynamic cluster formation in LEACH and by decreasing the number of transmissions and acceptance by using data aggregation. Although the clustering load is evade, PEGASIS still needs dynamic topology adaptation since a sensor node need to know about energy status of its neighbors to go to know where to route its data. Such topology adaptation can introduce significant load mostly for highly utilized networks.

\subsubsection{TEEN}

Threshold sensitive Energy Efficient sensor Network protocol (TEEN) [2] is a hierarchical protocol produced to be responsive to unexpected changes in the sensed attributes such as temperature. Responsiveness is urgent for time-critical applications, in which the network utilized in a reactive mode. TEEN chase a hierarchical approach along with the use of a data-centric process. The sensor network architecture is depended on a hierarchical grouping where near nodes form clusters and this mechanism goes on the second level until base station (sink) is reached. After the clusters are made, the cluster head broadcasts two thresholds to the nodes [2]. These are hard and soft thresholds for sensed attributes. The least possible value of an attribute to activate a sensor node to switch on its transmitter and transfer to the cluster head is hard threshold. Thus, the hard threshold permits the nodes to send only when the sensed attribute is in the range of regard, thus decreasing the number of transmissions significantly. Ever a node senses a value at or after the hard threshold, it send data only when the value of that attribute improve by an amount equal to or greater than the soft threshold. As a result, soft threshold will further minimize the number of transmissions if there is small or no change in the value of sensed attribute. One can adapt both hard and soft threshold values in order to control the number of package transmissions. However, TEEN is not fine for applications where regular reports are required since the user may not receive any data at all if the thresholds are not reached.

\subsubsection{TEEN-LCH}

TEEN-LCH a scalable and energy efficient routing protocol. In this protocol a new linear handling technique is used towards energy efficiency in linearly deployed WSNs with multiple static sinks in a linearly enhanced field [4]. The whole network field is separated into four equal sub regions .For efficient data gathering place three static sinks, i.e. two at the both corners sand one at the centre of the field. The protocol operations in divided into three different phases, Advertisement phase, Cluster setup phase, Data transmission phase. In this protocol the $\mathrm{CH}$ selection is based on the recommend percentage of $\mathrm{CHs}$ for the network and the number of times the node has been $\mathrm{CH}$ so far [4]. . TEENLCH shows more stability period as compared to that of TEEN.

\subsubsection{DEEC-LCH}

Same like as TEEN-LCH in this protocol use the linear cluster handling technique. In which the whole network is divided into different equal sub regions. For efficient data gathering place three static sinks, i.e. two at the both corners and one at the centre of the field. The protocol operations in divided into three different phases, Advertisement phase, Cluster setup phase, Data transmission phase. In this protocol the $\mathrm{CH}$ selection is based on the recommended percentage of $\mathrm{CHs}$ for the network and the number of times the node has been $\mathrm{CH}$ so far [4]. A proactive routing protocol Distributed Energy Efficient Clustering with Linear Cluster Handling (DEECLCH) [4] is implemented in this network field. In Comparison of DEEC-LCH and DEEC, networks lifetime of the proposed technique is increased. This is because those nodes save their energy due to formation of sub-region and placement of multiple sinks. [4]

\subsubsection{Adaptive Periodic Threshold Sensitive Energy Efficient Sensor Network Protocol (APTEEN)}

A new approach of TEEN is Adaptive Threshold sensitive Energy Efficient sensor Network protocol (APTEEN) that focuses at write down of periodic data collections and reacting time of critical events. The structure is alike to TEEN. In APTEEN after the Cluster Heads resolution takes place, it broadcasts the parameters like count time, attributes and threshold to all nodes. APTEEN's performance lies between LEACH and TEEN in terms of network lifetime and energy consumption. It makes an improvement over TEEN as it uses regular report for the time critical events. The main disadvantage is overhead and complexity of forming clusters of these two algorithms.

\subsubsection{Distributed Energy Efficient Clustering Algorithm for Heterogeneous Wireless Sensor Networks (DEEC)}

Advanced distributed multilevel clustering algorithm for heterogeneous WSN. In DEEC the cluster heads are chooses by a probability depends on the ratio between residual energy of each node and the average energy of the network. The approach of being cluster heads for nodes are dissimilar according to their initial and residual energy. The authors have supposed that all the nodes of the sensor network are provided with different amount of energy. Two levels of heterogeneous nodes are examined in the algorithm and after that a common result for multi-level heterogeneity is obtained. To avoid that every node require to know the global information of the networks, DEEC approximate the ideal value of network life-time, which is utilized to evaluate the reference energy that each node should spend during a round. Cluster calculates is variable in this approach lead to uneven clusters. 


\subsubsection{Hierarchical Energy Efficient Routing (HEERP)}

Hierarchical Energy Efficient Routing Protocol (HEERP) [5] for WSN establishes a new centralized approach to hierarchy formation. It does not examine the cluster formation and cluster head selection. The algorithm normally includes network hierarchy, neighbor table construction and data transmission. Here, the sink node begins the formation of hierarchy by broadcasting LCREQ packet. While, nodes left chooses LCREQ packets from nodes with fewer hop counts. Therefore, avoiding flooding of packets up to the time construction of the network. In data transmission stage, every node transmits data to their parent nodes. Node failure or battery consumption like factors is assumed in the maintenance stage. HEERP use less energy than the LEACH Protocol. Also simulation results show that delay in packet delivery is improved in HEERP in comparison to LEACH [5].

\subsubsection{HSEP}

Heterogeneity familiar with Hierarchical Stable Election Protocol (HSEP) for WSNs is a cluster based algorithm that presents a development over SEP. In SEP, heterogeneity of nodes is examined which uniformly consumes the extra amount of energy for advance nodes. Thus giving long lasting stability than LEACH. Separate threshold values for advanced and normal nodes are observed. HSEP is a heterogeneousaware clustering protocol that uses two types of $\mathrm{CHs}$ i.e. primary and secondary. The probability of nodes to become $\mathrm{CHs}$ is chooses by initial node energy that is relative to other network nodes. This approach provides a stability period. In HSEP, primary $\mathrm{CHs}$ check the distance among them and transmit the data to the secondary $\mathrm{CHs}$ (which are at minimum distance). TDMA approach is used for data transmission of nodes and the primary CHs. And also for primary and secondary $\mathrm{CHs}$ transmission. This improvement of HESP over SEP is due to two level hierarchy introductions.

\section{CONCLUSION}

In this generation the WSNs have very much enlarged performing an important part for the data efficient selection and their delivery. The energy efficiency is a very main issue for the networks mainly for WSNs which are described by limited battery ability. The intricacy and dependency of corporate operations on WSNs need the use of energyefficient routing procedure and protocols, which will assure the network connectivity and routing of information with the less demanded energy. In this paper, we focused on the energy efficient protocols that have been expended for WSNs. We classify the hierarchal routing Protocols, which name the data and query the nodes based on some element of the data are classified as data-centric. Many of the researchers obey this prototype in order to avoid the burden of making clusters, the use of specific nodes etc. Moreover, cluster-based routing protocols collect sensor nodes to useful broadcast the sensed information to the base station. The cluster heads are sometimes select as specific nodes that are less energyrequired. A cluster-head execute aggregation of sensed information and sends it to the base station in place of the nodes within its cluster. The more exciting research issue regarding such protocols is how to make the clusters so that the energy utilization and present communication measured such as latency are optimized. The element infecting cluster formation and cluster-head transmission are open issues for future research. Moreover, the process of sensed information and binding among clusters is also an exciting problem to investigate.

\section{ACKNOWLEDGMENTS}

The authors wish to thank the faculty from the computer science department at CTIEMT, Jalandhar for their continued support and feedback.

\section{REFERENCES}

[1] Jennifer Yick, Biswanath Mukherjee, Dipak Ghosal“ On the Wireless sensor network survey" ELSEVIER, Computer Networks 52 (2008) 2292-2330

[2] Kemal Akkaya, Mohamed Younis "A survey on routing protocols for wireless sensor networks" ELSEVIER,

[3] M.Shanthi Dr. E.RamaDevi "A Cluster Based Routing Protocol in Wireless Sensor Network for Energy Consumption" Int. J. Advanced Networking and Applications Volume: 05, Issue: 04, Pages:2015-2020 (2014) ISSN : 0975-0290

[4] M. Sajid, K. Khan, U. Qasim, Z.A. Khaan,S. Tariq, N. Javaid,"A New Linear Cluster Hnadling(LCH)Technique Toward's Energy efficiency in Linear WSNs" 2015 IEEE 29 International Conference on Advanced Information Networking and Applications.

[5] Parul Khurana, Inderdeep Aulakh Punjab University Chandigarh, India "Wireless Sensor Network Routing Protocols: A Survey" International Journal of Computer Applications Volume 75- No.15, August 2013.

[6] I.F. Akyildiz, W. Su*, Y. Sankarasubramaniam, E. Cayirci" Wireless sensors networks: a survey" ELSEVIER, Computer Networks 38 (2002) 393-422

[7] Jaspinder Kaur, Taranvir Kaur, Kanchan Kaushal "Survey on WSN Routing Protocols" International Journal of Computer Applications (0975 - 8887) Volume 109 - No. 10, January 2015

[8] Nikolaos A. Pantazis, Stefanos A. Nikolidakis and Dimitrios D.Vergados, Senior Member, IEEE" EnergyEfficient Routing Protocols in Wireless Sensor Networks: A Survey" IEEE communications survey \& tutorials, vol. 15, no. 2, second quarter 2013

[9] S.K. Singh, M.P. Singh, and D.K. Singh "Routing Protocols in Wireless Sensor Networks -A Survey" International Journal of Computer Science \& Engineering Survey (IJCSES) Vol.1, No.2, November 2010m DOI:

[10] Sanjeev Kumar Gupta, Neeraj Jain, Poonam Sinha “ Clustering Protocols in Wireless Sensor Networks: A Survey" International Journal of Applied Information Systems (IJAIS) - ISSN : 2249-0868 Foundation of Computer Science FCS, New York, USA Volume 5No.2, January 2013

[11] Shio Kumar Singh 1, M P Singh 2, and D K Singh 3 "Routing Protocols in Wireless Sensor Networks -A Survey" International Journal of Computer Science \& Engineering Survey (IJCSES) Vol.1, No.2, November 2010

[12] DaWei Xu, Jing Gao,a "Comparison Study to Hierarchical Routing Protocols in Wireless Sensor Networks" ELSEVIER Procedia Environmental Sciences 10 ( 2011 ) $595-600$

[13] B. Manzoor, N. Javaid, O. Rehman, M. Akbar, Q. Nadeem, A. Iqbal, M. Ishfaq" Q-LEACH: A New 
Routing Protocol for WSNs "Procedia Computer Science 19 ( 2013 ) 926 - 931

[14] S. Misra et al. (eds.), Guide to Wireless Sensor Networks, Computer Communications and Networks, DOI: 10.1007/978-1-84882-218-4 4, Springer-Verlag cAst2weszvxvx London Limited 2009.

[15] Ming Zhang, Yanhong Lu, Chenglong Gong, “ EnergyEfficient Routing Protocol based on Clustering and Least Spanning Tree in Wireless Sensor Networks", International Conference on Computer Science and Software Engineering,IEEE, 2008.
[16] Geetha. V., Pranesh.V. Kallapur, Sushma Tellajeera," Clustering in Wireless Sensor Networks: Performance Comparison of LEACH \& LEACH-C Protocols Using NS2” ELSEVIER Procedia Technology 4 ( 2012 ) 163 170

[17] F. Xiangning, S. Yulin. "Improvement on LEACH Protocol of Wireless Sensor Network", 2007, International Conference on Sensor Technologies and Applications pp 260-264, 